\title{
Economic Criteria in the Design of Cascades of CSTR's for the Performance of Enzyme-catalyzed Reactions
}

\author{
F. XAVIER MALCATA* \\ Escola Superior de Biotecnologia, Universidade Católica Portuguesa, Rua Dr. António Bernardino de Almeida, 4200 \\ Porto, Portugal
}

\begin{abstract}
The mass balances on substrate in each unit of a series of CSTRs performing an enzyme-catalyzed reaction described by Michaelis-Menten kinetics (with parameter $K_{m}$ ) are written and the necessary and sufficient condition that must be satisfied by the intermediate concentrations in order to obtain a minimum overall capital investment is found on the assumption that the cost of each reactor unit scales up on its capacity according to a fractional factor exponential rule (with parameter $n$ ). The asymptotic situations of pseudo-zero order and pseudo-first order behavior are explored. The ratio between consecutive concentrations leading to a minimum overall capital investment decreases as $K_{m}$ decreases at a rate that is slower for higher $n$, and tends to unity as the pseudo-first order situation is approached. If fractional values of $n$ are considered, local minima of the capital investment associated with the overall reactor cascade exist only in certain ranges of substrate conversion; below the lower limits of such ranges, the number of reactor units should actually be decreased. A graphical procedure aimed at obtaining the intermediate optimal concentrations is presented.
\end{abstract}

\begin{abstract}
On a établi les bilans massiques sur substrat dans chaque unité d'une série de réacteurs à réservoir agité continus dans le cadre d'une réaction catalysée aux enzymes décrite par les cinétiques de Michaelis-Menten (avec le paramètre $K_{m}$ ). On a trouvé la condition nécessaire et suffisante que doivent remplir les concentrations intermédiaires pour un investissement en capital global minimum avec comme hypothèse que le coût de chaque réacteur augmente proportionnellement avec sa capacitié selon une règle exponentielle de facteurs fractionnels (avec le paramètre $n$ ). Les situations asymptotiques de comportement de l'ordre pseudo-zéro et pseudo-premier ordre sont étudiées. Le rapport entre les concentrations consécutives conduisant à un investissement en capital global minimum diminue lorsque $K_{m}$ diminue à une vitesse inférieure pour les grandes valeurs de $n$, et il tend vers l'unité lorsqu'on s'approche de la situation pseudo-premier ordre. Pour des valeurs fractionnelles de $n$, des minimums locaux de l'investissement en capital en rapport avec la cascade globale de réacteurs existent seulement dans certaines gammes de conversion du substrat: en-dessous des limites inférieures de ces gammes, le nombre de réacteurs devrait être effectivement réduit. On présente une méthode graphique permettant d'obtenir les concentrations optimales intermédiaires.
\end{abstract}

Keywords: capital investment, exponential factor rule, Michaelis-Menten reactions, optimization.

$\mathrm{C}$ ascades of CSTRs have for decades received the attention of several researchers aiming at the performance of enzyme-catalyzed chemical reactions which require uniformity of temperature, which can be brought about by crude preparations of enzyme, and for which thermal processing of the effluent streams is a standard practice. An example of potential industrial application is the production of low-lactose skim milk for lactose-intolerant consumers using a food-grade $\beta$-galactosidase of microbial origin in free form that hydrolyzes lactose into their monosaccharide subunits, glucose and galactose. From the pioneering work by Aris (1961) and Levenspiel (1972) encompassing reactor design and optimization for several constant $n$-th order kinetics, optimal designs of CSTR cascades using as the objective function minimization of the overall reactor volume have been obtained for irreversible (Luyben and Tramper, 1982) and reversible (Malcata and Cameron, 1992) MichaelisMenten kinetics, Ping-Pong Bi-Bi kinetics (Malcata, 1988) and Hill's asymptotic multisited rate expression (Malcata, 1989a), all under the assumption that such reactions are catalyzed by soluble enzymes in the complete absence of deactivation. Later, the cases of Michaelis-Menten irreversible kinetics coupled with first order irreversible deactivation under isothermal (Lopes and Malcata, 1993) and nonisothermal (Paiva and Malcata, 1993) conditions have been also worked out. The actual departure from optimality which arises when a constant-volume cascade is employed instead

"E-mail address: xmalcata@esb.ucp.pt of a minimum-volume cascade was also theoretically discussed (Malcata, 1995a) for Michaelis-Menten irreversible kinetics. However, such volumetric objective functions do not approach the actual minimum capital investment on equipment, which proves to be a major constraint in industrial scenarios. Such difficulty was somewhat overcome elsewhere (Malcata, 1989b) via the assumption that cost scaleup is adequately described by a power rule on the equipment capacity with an exponential factor greater than unity and by combining cost scaleup factors with downstream separation costs (Malcata, 1995b).

This communication addresses the issue of minimum overall reactor cost in the more realistic situation where the value of the aforementioned exponent for cost scaleup lies between zero and unity for reactions described by irreversible Michaelis-Menten kinetics and carried out in a cascade of CSTRs under the assumption that the cost of the final product is controlled by the capital investment associated with the reactor network.

\section{Theoretical analysis}

Assuming a reaction described by the chemical equation $\mathrm{S} \rightarrow$ products (where $\mathrm{S}$ denotes substrate) which is carried on in a cascade of CSTRs, the mass balance on $S$ can be written as:

$$
D a_{i}=\frac{\left(C_{i-1}^{*}-C_{i}^{*}\right)\left(K_{m}^{*}+C_{i}^{*}\right)}{C_{i}^{*}}, i=1,2, \ldots, N \ldots \ldots
$$


where the normalized concentration of $S$ is defined as $C_{i}^{*}=$ $C_{1} / C_{0}$, the dimensionless Michaelis-Menten parameter as $K_{m}^{*}=K_{m} / C_{0}$, the Damköhler number as $D a_{i}=v_{\max } V \hat{i} / C_{0} Q$, and where $C_{i}$ denotes the substrate concentration at the outlet of the $i$-th reactor, $C_{0}$ the inlet substrate concentration to the first reactor, $V_{i}$ the volume of the $i$-th reactor, $Q$ the volumetric flow rate through the cascade, $K_{m}$ the Michaelis-Menten parameter, and $v_{\max }$ the rate of the enzyme-catalyzed reaction under saturation of substrate. (Obviously $C_{0}^{*}=1$ ).

Assuming that the capital investment associated with the $i$-th reactor unit, $I_{i}$, is calculated via the approximate empirical relationship:

$$
I_{i}=\alpha V_{i}^{n}, i=1,2, \ldots, N
$$

where $\alpha$ and $n$ denote constants, then, in view of the variables defined previously, the necessary and sufficient condition for a minimum of capital investment associated with the CSTR cascade can be written as:

$$
\frac{\partial\left\{\sum_{j=1}^{N} D a_{j}^{n}\right\}}{\partial C_{i}^{*}}=0, i=1,2, \ldots, N-1
$$

coupled with:

$$
\frac{\partial^{2}\left\{\sum_{j=1}^{N} D a_{j}^{n}\right\}}{\partial C_{i}^{* 2}}>0, i=1,2, \ldots, N-1 \ldots \ldots \ldots \ldots
$$

Recalling Equation (1), $D a_{j}=D a_{j}\left\{K_{m}^{*}, C_{j-1}^{*}, C_{j}^{*}\right\}$; hence, Equations (3)-(4) may be simplified to (Luyben and Tramper, 1982):

$$
\frac{\partial\left\{D a_{i}{ }^{n}+D a_{i+1}{ }^{n}\right\}}{\partial C_{i}^{*}}>0, i=1,2, \ldots, N-1 \ldots \ldots \ldots
$$

and

$$
\frac{\partial^{2}\left\{D a_{i}^{n}+D a_{i+1}{ }^{n}\right\}}{\partial C_{i}^{* 2}}>0, i=1,2, \ldots, N-1
$$

respectively. Combination of Equations (1), (5), and (6) leads, after some algebraic rearrangement, to:

$$
\begin{aligned}
& \frac{C_{i}^{* 2}+K_{m}^{*} C_{i-1}^{*}}{C_{i}^{* 2}}\left(\frac{\left(C_{i-1}^{*}-C_{i}^{*}\right)\left(K_{m}^{*}+C_{i}^{*}\right)}{C_{i}^{*}}\right)^{n-1} \\
& -\frac{K_{m}^{*}+C_{i+1}^{*}}{C_{i+1}^{*}}\left(\frac{\left(C_{i}^{*}-C_{i+1}^{*}\right)\left(K_{m}^{*}+C_{i+1}^{*}\right)}{C_{i+1}^{*}}\right)^{n-1} \\
& \quad=0, i=1,2, \ldots, N-1 \ldots \ldots \ldots
\end{aligned}
$$

for $n \neq 0$, and

$$
\begin{gathered}
n(n-1)\left(\frac{C_{i}^{* 2}+K_{m}^{*} C_{i-1}^{*}}{C_{i}^{* 2}}\right)^{2}\left(\frac{\left(C_{i-1}^{*}-C_{i}^{*}\right)\left(K_{m}^{*}+C_{i}^{*}\right)}{C_{i}^{*}}\right)^{n-2} \\
+\frac{2 n K_{m}^{*} C_{i-1}^{*}}{C_{i}^{* 3}}\left(\frac{\left(C_{i-1}^{*}-C_{i}^{*}\right)\left(K_{m}^{*}+C_{i}^{*}\right)}{C_{i}^{*}}\right)^{n-1} \\
+n(n-1)\left(\frac{K_{m}^{*}+C_{i+1}^{*}}{C_{i+1}^{*}}\right)^{2}\left(\frac{\left(C_{i}^{*}-C_{i+1}^{*}\right)\left(K_{m}^{*}+C_{i+1}^{*}\right)}{C_{i+1}^{*}}\right)^{n-2} \\
>0, i=1,2, \ldots, N-1
\end{gathered}
$$

Equations (7) and (8) may be further rearranged to yield Equations (9) and (10), respectively, viz.:

$$
\begin{aligned}
& \left(\frac{\frac{C_{i+1}^{*}}{C_{i}^{*}}\left(1-\frac{C_{i}^{*}}{C_{i-1}^{*}}\right)\left(1+\frac{K_{m}^{*}}{C_{i}^{*}}\right)}{\left.\frac{C_{i}^{*}}{C_{i-1}^{*}}\left(1-\frac{C_{i+1}^{*}}{C_{i}^{*}}\right)\left(\frac{K_{m}^{*}}{C_{i}^{*}}+\frac{C_{i+1}^{*}}{C_{i}^{*}}\right)\right)^{n-1}}\right. \\
& =\frac{\frac{C_{i}^{*}}{C_{i-1}^{*}}\left(\frac{K_{m}^{*}}{C_{i}^{*}}+\frac{C_{i+1}^{*}}{C_{i}^{*}}\right)}{\frac{C_{i+1}^{*}}{C_{i}^{*}}\left(\frac{K_{m}^{*}}{C_{i}^{*}}+\frac{C_{i}^{*}}{C_{i-1}^{*}}\right)}, i=1,2, \ldots, N-1
\end{aligned}
$$

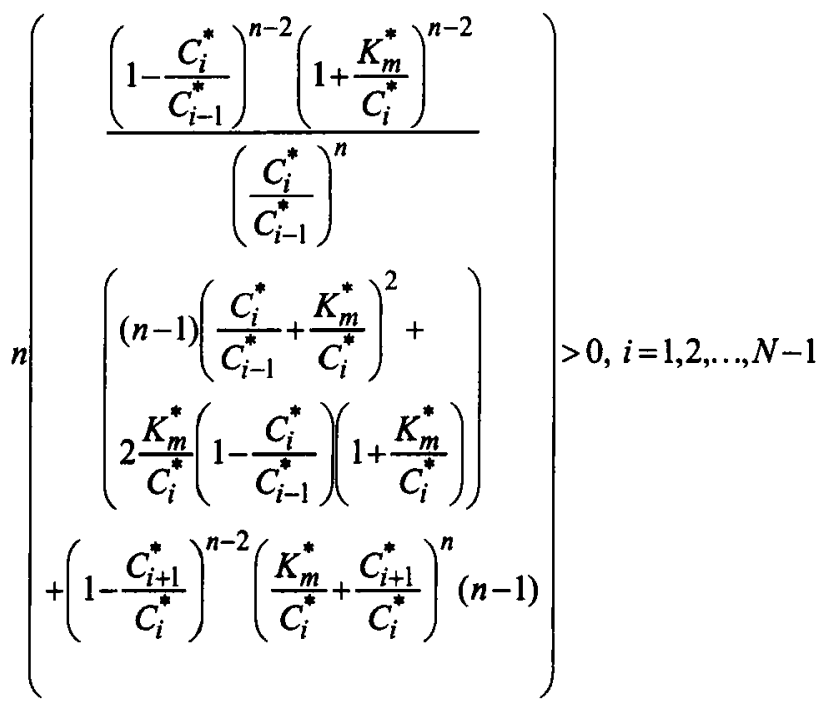

Plots of the intermediate concentrations which lead to minima of the overall reactor investment as given by Equations (9) and (10) are available in Figure I for several values of parameter $K_{m}^{*} / C_{i}^{*}$; these plots were prepared via solution of Equation (9) using Newton's iterative method with initial estimates of $0.99 C_{i}^{*} / C_{i-1}^{*}$ (convergence to a final error below 0.0001 was achieved in no more than ten iterations). Plots of the second derivative as given by Equation (10) calculated at the aforementioned loci of optima for a selected fractional value of $n$ and several values of $K_{m}^{*} / C_{i}^{*}$ are available in Figure 2. 

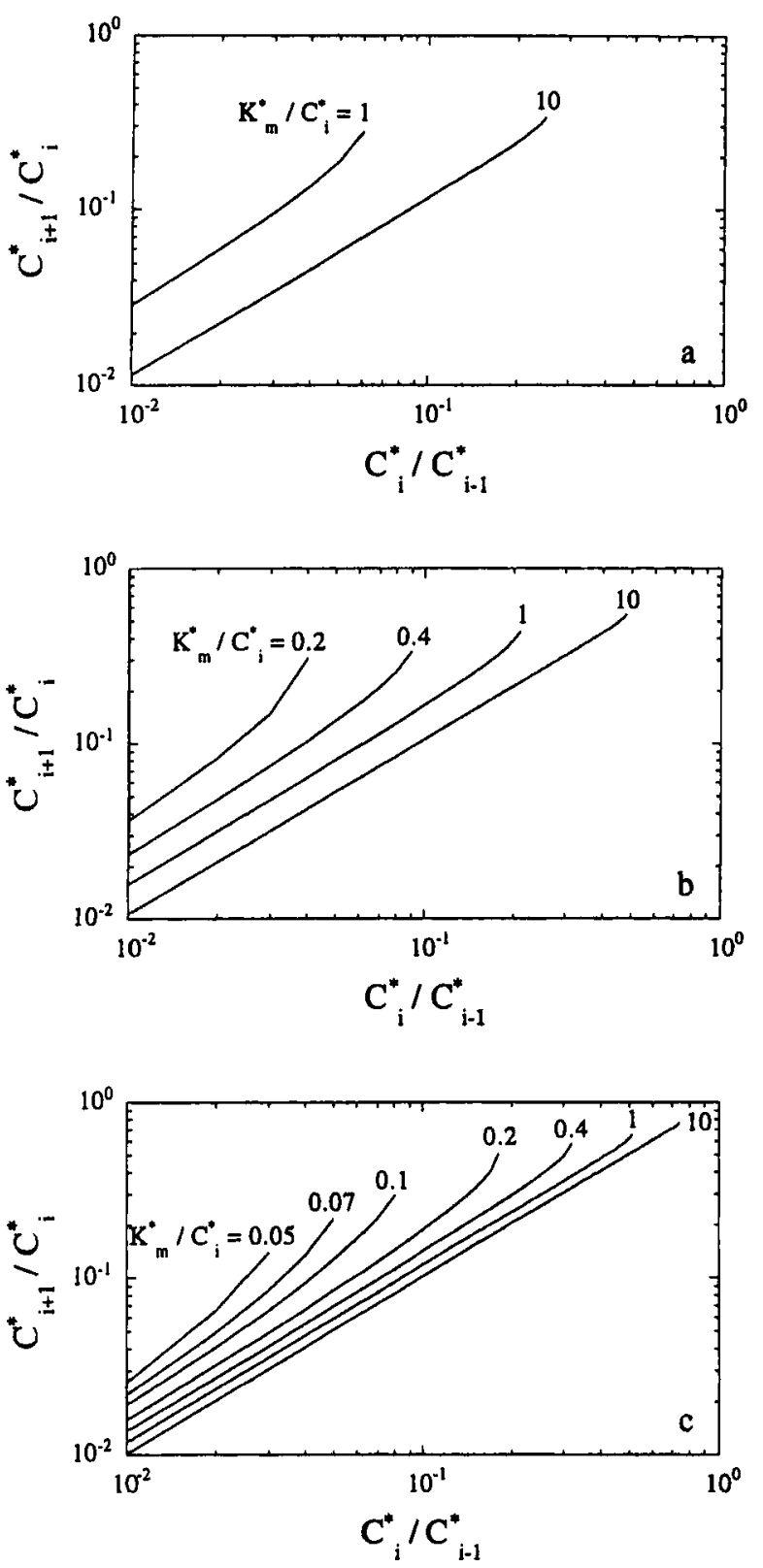

Figure $1-$ Loci of the intermediate concentrations of substrate which yield a minimum overall volume in the cascade of CSTRs for a given conversion under the assumption of a MichaelisMenten reaction plotted as $C_{i+1}^{*} / C_{i}^{*}$ vs. $C_{i}^{*} / C_{i-1}^{*}$ for several values of the ratio $K_{m}^{*} / C_{i}^{*}$ and for (a) $n=0.4$, (b) $n=0.6$, and (c) $n=0.8$.

In the case of the asymptotic behavior of pseudo-zero order (i.e. when $K_{m}^{*} \ll 1$ ), Equations (7) and (8) become:

$$
C_{i}^{*}=\frac{C_{i-1}^{*}+C_{i+1}^{*}}{2}, i=1,2, \ldots, N-1
$$

for $n \neq 0$, and

$$
\begin{gathered}
n(n-1)\left(C_{i-1}^{*}-C_{i}^{*}\right)^{n-2}+n(n-1)\left(C_{i}^{*}-C_{i+1}^{*}\right)^{n-2}>0, \\
\quad i=1,2, \ldots, N-1 \ldots \ldots \ldots \ldots \ldots \ldots \ldots \ldots \ldots
\end{gathered}
$$

respectively. Equation (12) may be arranged to yield:

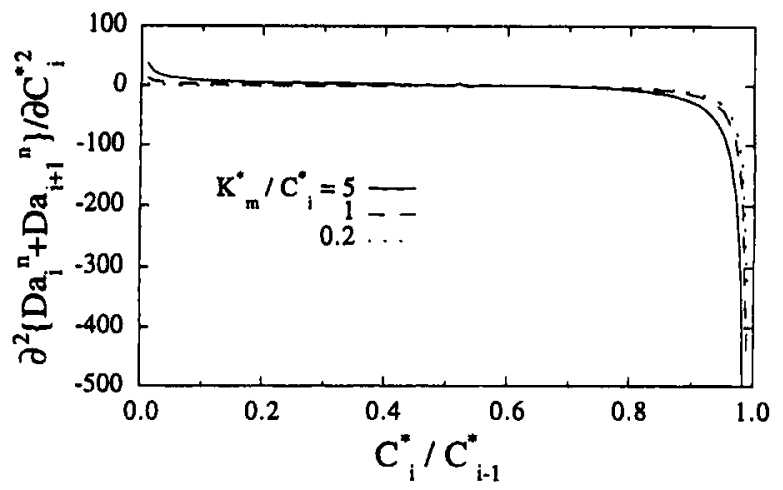

Figure 2 - Variation of $\partial^{2}\left\{D a_{i}^{n}+D a_{i+1}{ }^{n}\right\} / \partial C_{i}^{* 2}$ evaluated at the loci of intermediate concentrations that lead to a nul value of the corresponding first derivative plotted vs. $C_{i}^{*} / C_{i-1}^{*}$ for $n=0.6$ and several values of the ratio $K_{m}^{*} / C_{i}^{*}$.

$$
\begin{gathered}
n(n-1)\left(\left(\frac{1-\frac{C_{i}^{*}}{C_{i-1}^{*}}}{\frac{C_{i}^{*}}{C_{i-1}^{*}}}\right)^{n-2}+\left(1-\frac{C_{i+1}^{*}}{C_{i}^{*}}\right)^{n-2}\right) \\
>0, i=1,2, \ldots, N-1 \ldots \ldots \ldots
\end{gathered}
$$

In the case of the asymptotic behavior of pseudo-first order (i.e. when $K_{m}^{*} \gg>1$ ), Equations (7) and (8) become:

$$
\left(\frac{\frac{C_{i+1}^{*}}{C_{i}^{*}}\left(1-\frac{C_{i}^{*}}{C_{i-1}^{*}}\right)}{\frac{C_{i}^{*}}{C_{i-1}^{*}}\left(1-\frac{C_{i+1}^{*}}{C_{i}^{*}}\right)}\right)^{n-1}=\frac{\frac{C_{i}^{*}}{C_{i-1}^{*}}}{\frac{C_{i+1}^{*}}{C_{i}^{*}}}, i=1,2, \ldots, N-1 \ldots
$$

for $n \neq 0$, and

$$
\begin{gathered}
n(n-1)\left(\frac{K_{m}^{*} C_{i-1}^{*}}{C_{i}^{* 2}}\right)^{2}\left(\frac{K_{m}^{*}\left(C_{i-1}^{*}-C_{i}^{*}\right)}{C_{i}^{*}}\right)^{n-2} \\
+\frac{2 n K_{m}^{*} C_{i-1}^{*}}{C_{i}^{* 3}}\left(\frac{K_{m}^{*}\left(C_{i-1}^{*}-C_{i}^{*}\right)}{C_{i}^{*}}\right)^{n-1} \\
+n(n-1)\left(\frac{K_{m}^{*}}{C_{i+1}^{*}}\right)^{2}\left(\frac{K_{m}^{*}\left(C_{i}^{*}-C_{i+1}^{*}\right)}{C_{i+1}^{*}}\right)^{n-2} \\
>0, i=1,2, \ldots, N-1 \ldots \ldots
\end{gathered}
$$

respectively. Equation (15) may be further rearranged to give:

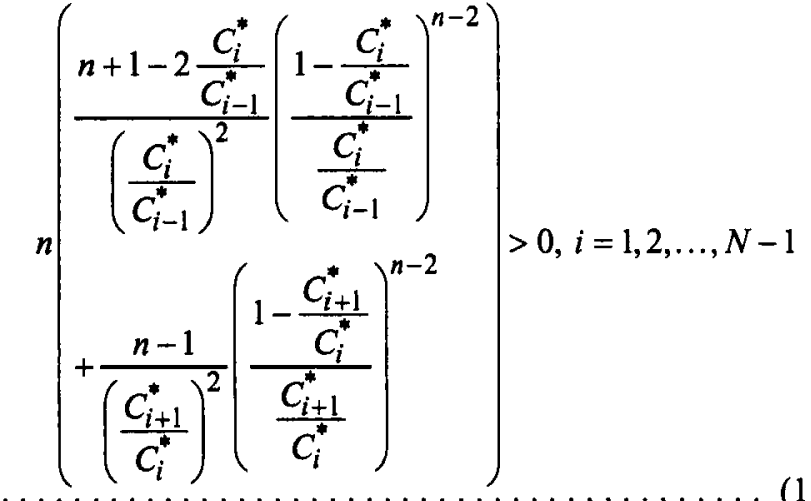


respectively. It is apparent from Equation (16) that the necessary and sufficient condition for the minimum reduces to:

$$
C_{i}^{*}=\sqrt{C_{i-1}^{*} C_{i+1}^{*}}<n
$$

Recalling Equation (2), the normalized capital investment corresponding to each CSTR unit may be given by:

$$
I_{i}^{*}=\left(\frac{D a_{i}^{*}}{D a_{1,1}^{*}}\right)^{n}, i=1,2, \ldots, N
$$

where $I_{i}^{*}$ is defined as $I_{i} I_{1,1}$, and $I_{1,1}$ and $D a_{1,1}$ denote the investment required by and the Damköhler number associated with a single CSTR able to perform the specified conversion, viz. $1-C_{N}^{*}$. The total investment, $I_{t o r}^{*}$ is then:

$$
I_{t o t}^{*}=\sum_{i=1}^{N}\left(\frac{C_{N}^{*}}{C_{i}^{*}} \frac{C_{i-1}^{*}-C_{i}^{*}}{1-C_{N}^{*}} \frac{K_{m}^{*}+C_{i}^{*}}{K_{m}^{*}+C_{N}^{*}}\right)^{n} \ldots \ldots \ldots
$$

In the case of the asymptotic first order behavior, then application of Equation (17) consecutively from $i=1$ up to a generic $i$ yields:

$$
C_{i}^{*}=C_{i+1}^{*}{ }^{i /(i+1)}
$$

Application of Equation (20) consecutively from $i=N$ down to a generic $i$ yields:

$$
C_{i}^{*}=C_{N}^{* i / N}
$$

Combination of Equations (1) and (21) finally gives:

$$
D a_{i, o p t}=K_{m}^{*}\left(C_{N}^{*-1 / N}-1\right), i=1,2, \ldots, N \ldots \ldots \ldots
$$

Recalling Equation (2), the normalized capital investment corresponding to each CSTR unit will thus be given by:

$$
I_{i}^{*}=\left(\frac{C_{N}^{*-1 / N}-1}{C_{N}^{*-1}-1}\right)^{n}, i=1,2, \ldots, N \ldots \ldots \ldots \ldots
$$

and the total minimum investment will be:

$$
I_{t o t, \min }^{*}=N\left(\frac{C_{N}^{*}(N-1) / N}{1-C_{N}^{*}}\right)^{*}
$$

Equation (24) is graphically plotted in Figure 3. Using Hôpital's rule, one finally concludes that the asymptotic limit of Equation (24) when $N$ tends to infinity is given by:

$$
I_{\text {tot } \text { min }}^{*}=\frac{C_{N}^{*}}{1-C_{N}^{*}} \ln \left\{\frac{1}{C_{N}^{*}}\right\} \ldots \ldots \ldots \ldots \ldots \ldots
$$

when $n=1$, and $\Gamma_{\text {tot, min }}^{*}=\infty$ when $0<n<1$.

\section{Discussion}

Assurance that the critical point computed by setting the first derivative equal to zero describes a minimum is rather important throughout the analysis, and is obtained at the
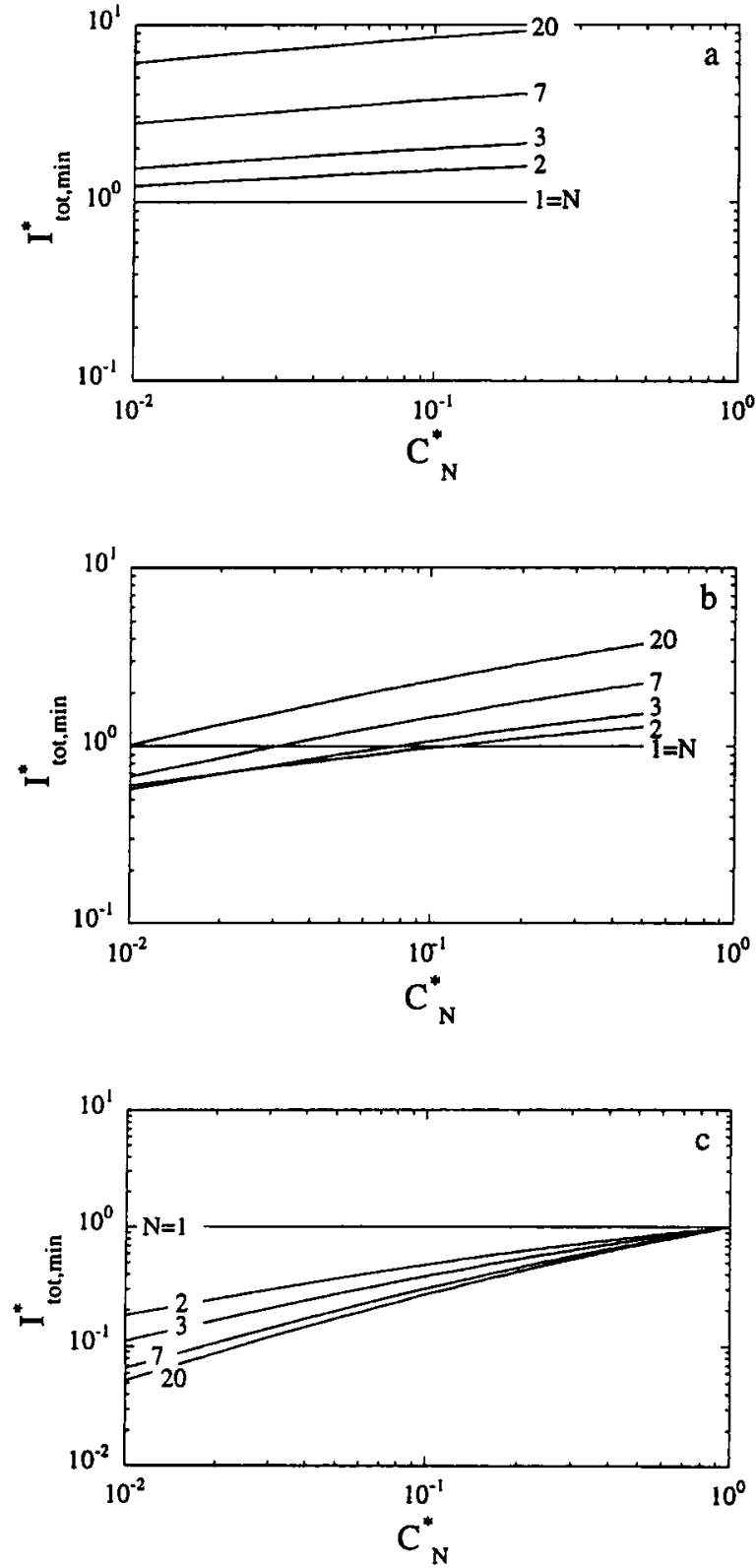

Figure 3-Loci of the intermediate minimum capital investments associated with the cascade of CSTRs for a given conversion and a given number of reactors, $N$, under the assumption of a pseudofirst order reaction plotted as $I_{\text {tot, } \min }^{*}$ vs. $C_{N}^{*}$ for (a) $n=0.2$, (b) $n=$ 0.5 , and (c) $n=1$.

expense of setting the second derivative above zero. Inspection of Equation (10) coupled with the implicit physical constraint $1 \geq C_{i-1}^{*}>C_{i}^{*}>0$ (for $i=1,2, \ldots, N$ ) indicates that the critical point calculated via Equation (9) for typical Michaelis-Menten behavior is a minimum whenever $n<0$ or $n>1$; this conclusion confirms previous work by Malcata (1989b) for $n>1$. By the same token, inspection of Equation (13) indicates that the critical point calculated via Equation (11) for pseudo-zero order behavior is a minimum whenever $n$ $<0$ or $n>1$. Finally, inspection of Equation (16) indicates that the critical point calculated via Equation (14) for pseudo-first order behavior is a minimum whenever $n<0$ or $n>1$. When $n$ takes fractional values, then decision on whether the point in question is a minimum or not becomes a nontrivial task and numerical work is in general required. 
It is interesting to note that not all outlet concentrations of substrate allow minima to be obtained for a given cost exponential factor, a given cascade of CSTRs, and a given set of kinetic parameters. This is the reason why the curves in Figure 1 were discontinued at some point in the $C_{i+1}^{*} / C_{i}^{*} / C_{i-1}^{*}$ domain; after the aforementioned point, the curves can be drawn smoothly but give rise to local maxima instead (as confirmed by the negative sign of the second derivative, as apparent from inspection of Figure 2). Such point of discontinuity occurs earlier when $K_{m}^{*} / C_{i}^{*}$ is smaller; hence, when $K_{m}^{*}$ tends to zero (i.e. when the pseudo-zero order behavior is approached) no curve can be drawn at all (or, equivalently, the slope of the curve becomes infinity), whereas when $K_{m}^{*}$ grows without limit (i.e. when the pseudofirst order behavior is approached) the curve coincides with the main diagonal. In the latter limiting case, the necessary condition for an optimum as set forth by Equation (14) leads to a minimum when $\left(C_{i}^{*} / C_{i-1}^{*}\right) \leq n$ and to a maximum when $\left(C_{i}^{*} / C_{i-1}^{*}\right)>n$. The slope of the curve is also approximately constant and equal to unity except in the vicinity of the point of discontinuity. Furthermore, the rate of displacement of the curve with $K_{m}^{*} / C_{i}^{*}$ decreases as $n$ increases. When no local minimum can be found (i.e. when $C_{i}^{*} / C_{i-1}^{*}$ is too high), then the global minimum lies on a physicochemical restriction, viz. $\left(C_{i+1}^{*} / C_{i}^{*}\right)=1$; this condition is equivalent to saying that the reactor in question has zero volume or, alternatively, that the reactor should be eliminated from the cascade.

Careful inspection of Figure 1 also indicates that higher values for $K_{m}^{*} / C_{i}^{*}$ lead to higher values for the ratio of $C_{i+1}^{*} / C_{i}^{*}$ to $C_{i}^{*} /_{i-1}^{*}$, and that such ratio is above unity irrespective of the value of $K_{m}^{*}$; when $n=1$ and $K_{m}^{*}$ is very large, then the aforementioned ratio is exactly unity irrespective of the value of $K_{m}^{*}$. These two observations imply that the optimum intermediate concentration is always below the geometric mean of the consecutive prior and posterior concentrations except in the case where $n=1$ for which it is equal to the geometric mean. These findings are in good agreement with Luyben and Tramper (1982), who claimed that when the objective function is minimization of the overall reactor cascade volume (rather than the overall reactor cost) then $C_{i}^{*}=\sqrt{C_{i-1}^{*} C_{i+1}^{*}}$.

Although utilization of Equation (19) allows calculation of the overall cost of the reactor network for a given final conversion, discussion of the behavior of $I_{t o t, \min }^{*}$ with the important variables is simpler for the asymptotic case of large $K_{m}^{*}$, and was consequently depicted in Figure 3. It is apparent that the curves representing $I_{t o t, \text { min }}^{*}$ become closer and closer with increasing $n$ at the upper limit of concentration allowed for a feasible minimum and eventually collapse when $C_{N}^{*}=n=1$; on the other hand, when $C_{N}^{*}$ increases, then $I_{t o t, \min }^{*}$ also increases, thus suggesting that a cascade of CSTRs becomes better than a single CSTR in terms of cost when higher conversions are sought; finally, there is a threshold for the number of units in the reactor cascade able to perform a given conversion if a lower cost than that of a single CSTR is to be obtained, which occurs at lower $C_{N}^{*}$ as $n$ decreases (see e.g. Figure $3 \mathrm{~b}$ : for $C_{N}^{*}=0.01$ the minimum overall cost is actually obtained when between 2 and 3 reactors are used in the cascade). As expected, when $N$ tends to infinity then $I_{t o t \text {, min }}^{*}$ tends to a finite limit given by the ratio of size of a plug flow reactor able to perform the same conversion as single CSTR to the size of such CSTR.

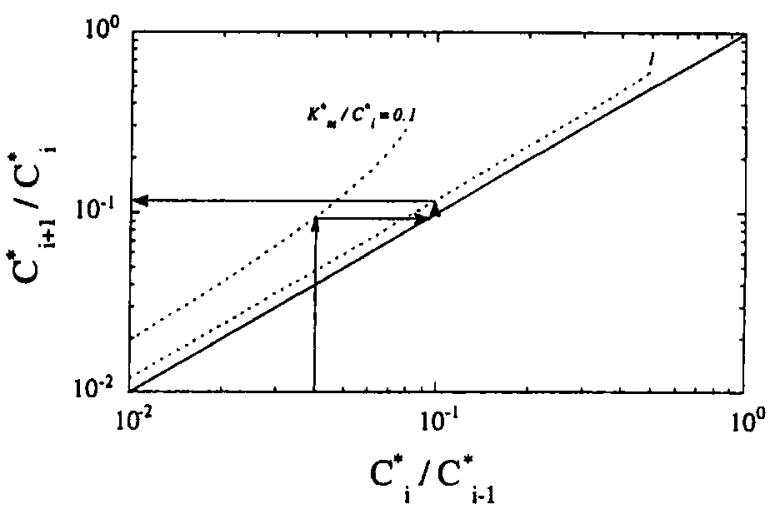

Figure 4-Example of a graphical determination of intermediate ratios of substrate concentrations that lead to a minimum overall cascade cost.

\section{Numerical example}

Consider that $n=0.8, C_{N}^{*}=0.00048, K_{m}^{*}=0.004$, and $N$ $=3$. Recalling Figure 1c, one may arbitrarily choose a value for the initial ratio $C_{1}^{*} / C_{0}^{*}$, say 0.04 , and proceed vertically from that abcissa up to the curve corresponding to $K_{m}^{*} / C_{1}^{*}=$ 0.1 , as indicated by the first arrow in Figure 4; the ordinate of this point on the curve, i.e. 0.1 , then gives the value for the ratio $C_{2}^{*} / C_{1}^{*}$ (or, equivalently, $C_{2}^{*}=0.004$ )). The aforementioned procedure must then be repeated in order to obtain $C_{3}^{*} / C_{2}^{*}$ from $C_{2}^{*} / C_{1}^{*}$ using again Figure 1c; this corresponds geometrically to proceed horizontally in Figure 4 from the ordinate $C_{2}^{*} / C_{1}^{*}=0.1$ right to the main diagonal (in bold), as indicated by the second arrow, and to proceed vertically from that point (which has $C_{2}^{*} / C_{1}^{*}=0.1$ as abcissa) up to the curve corresponding to $K_{m}^{*} / C_{2}^{*}=1$, as indicated by the third arrow. From that point one may finally proceed horizontally left to the vertical axis, as indicated by the fourth arrow, which corresponds to ordinate $C_{3}^{*} / C_{2}^{*}=0.12$, or, equivalently, $C_{3}^{*}=0.00048$. Using these data in Equation (19), one finally gets 0.206 for $I_{\text {tor }}^{*}$. If the graphical procedure did not yield the desired value of conversion, then the whole process would have to be repeated from another starting estimate of $C_{1}^{*} / C_{0}^{*}$. (Although the parameter values chosen for the numerical example may seem, at least partially, as devoid of practical interest, it should be noted that they were chosen to illustrate the graphical methodology, and so clarity was deliberately given a higher priority than applicability).

\section{Nomenclature}

$C_{i}=$ substrate concentration in the $i$-th reactor $\left(\mathrm{mol} / \mathrm{m}^{3}\right)$

$C_{i}^{*} \quad=$ normalized counterpart of $C_{i}, C_{i}^{*}=C_{i} / C_{0}(-)$

$C_{0}=$ substrate concentration at the inlet to the 1 st reactor $\left(\mathrm{mol} / \mathrm{m}^{3}\right)$

$D a_{i}=$ Damköhler number associated with the $i$-th reactor, $D a_{i}=$ $v_{\max } V_{i} / C_{0} Q(-)$

$D a_{1,1}=$ Damköhler number associated with the reactor of a single-unit cascade, $D a_{1,1}=v_{\max } V_{1} / C_{0} Q(-)$

$I_{1,1}=$ capital investment associated with the reactor of a single-unit cascade (\$)

$I_{i} \quad=$ capital investment associated with the $i$-th reactor (\$)

$I_{i}^{*} \quad=$ normalized capital investment associated with the $i$-th reactor, $I_{i} / I_{1,1}(-)$

$I_{t o t}^{*} \quad=$ normalized capital investment associated with the overall cascade $(-)$ 
$I_{t o t, \text { min }}=$ normalized minimum capital investment associated with the overall cascade $(-)$

$K_{m} \quad=$ Michaelis-Menten parameter $\left(\mathrm{mol} / \mathrm{m}^{3}\right)$

$K_{m}^{*}=$ normalized Michaelis-Menten parameter, $K_{m}^{*}=K_{m} / C_{0}(-)$

$n \quad=$ exponential constant $(-)$

$N \quad=$ number of reactors in the cascade $(-)$

$Q=$ volumetric flow rate through the cascade $\left(\mathrm{mol} / \mathrm{m}^{3}\right)$

$S=$ substrate

$V_{i} \quad=$ volume of the $i$-th reactor $\left(\mathrm{m}^{3}\right)$

$v_{\max }=$ rate of enzyme-catalyzed reaction under saturation of substrate $\left(\mathrm{mol} / \mathrm{m}^{3} . \mathrm{s}\right)$

\section{Greek letter}

$\alpha \quad=$ proportionality constant $\left(\$ / \mathrm{m}^{3 \mathrm{n}}\right)$

\section{References}

Aris, R., "The Optimal Design of Chemical Reactors", Academic Press, New York (1961).

Levenspiel, O., "Chemical Reaction Engineering", John Wiley \& Sons, New York (1972).

Lopes, T. I. and F. X. Malcata, "Optimal Design of a Series of CSTR's for Biochemical Reactions in the Presence of Enzyme Deactivation", J. Chem. Eng. Japan 26, 94-98 (1993).

Luyben, K. C. and J. Tramper, "Optimal Design for Continuous Stirred Tank Reactors in Series Using Michaelis-Menten Kinetics", Biotechnol. Bioeng. 24, 1217-1220 (1982).
Malcata, F. X., "Optimal Design on an Economic Basis for Continuous Stirred Tank Reactors in Series Using MichaelisMenten Kinetics for Ping-Pong Reactions", Can. J. Chem. Eng. 66, 168-172 (1988).

Malcata, F. X., "CSTR's in Biochemical Reactions - An Optimization Problem", Chem. Eng. Ed. 23, 112-115, 128 (1989a).

Malcata, F. X., "A Heuristic Approach for the Economic Optimization of a Series of CSTRs Performing Michaelis-Menten Reactions", Biotechnol. Bioeng. 33, 25 I-255 (1989b).

Malcata, F. X. and D. C. Cameron, "Optimal Design of a Series of CSTR's Performing Reversible Reactions Catalyzed by Soluble Enzymes: A Theoretical Study", Biocatalysis 5, 233-248 (1992).

Malcata, F. X., "To What Extent is a Constant Volume Design Worse than a Minimum Volume Design for a Series of CSTR's?", Bioprocess Eng. 13, 105-107 (1995a).

Malcata, F. X., "Cost Minimization in the Predesign of an Enzymatic CSTR: An Overall Approach", Bioprocess Eng. 13, 51-55 (1995b).

Paiva, A. L. and F. X. Malcata, "Optimal Temperature and Concentration Profiles in a Cascade of CSTR's Performing Michaelis-Menten Reactions with First Order Enzyme Deactivation", Bioprocess Eng. 9, 77-82 (1993). 